\title{
The Ministers
}

MARIANA IBANEZ

Harvard University

CHRISTINA JAMES

University of Calgary

The Ministers are luminous lightweight spheroids, self-supporting monocoque shells that anchor a festival devoted to the co-mingling of design and science. They offer a meaningful if not easily described interactivity. They are the constituent parts of a Ministry that listens without judgment. They receive the secrets of visitors and function as open and democratic figures within a collective dedicated to the production of culture.

The two figures included here, Sputnik and Nancy, offer two surfacing approaches for the production of thin shell structures. In the case of Sputnik creased edges allow extremely thin aluminum sheets to hold their form and produce a series of light emitting serrations. Nancy deploys a series of translucent interlocking doubly curved surfaces. The edges are joined with rivets that express a secondary pattern on the continuous surfaces.

Deployed as large scale light beacons on the festival grounds the objects elicit interaction from the public through their intrinsic qualities. Sputnik as a reflective surface mirroring the activities of the crowds and events surrounding it, and Nancy as a glowing vessel into which participants insert their hands, faces and cameras to explore its interiority.

These projects are the result of design collaborations between the Laboratory for Integrative Design at the University of Calgary, and Ibañez Kim Studio. Over the course of 3 months a series of digital protocols and
JASON S. JOHNSON

University of Calgary

LOGAN ARMSTRONG

University of Calgary
MATTHEW PARKER

University of Calgary

SIMON KIM

University of Pennsylvania physical prototypes were developed in tandem to gain control over the precision with which the large scale objects could be visualized, fabricated and assembled. 


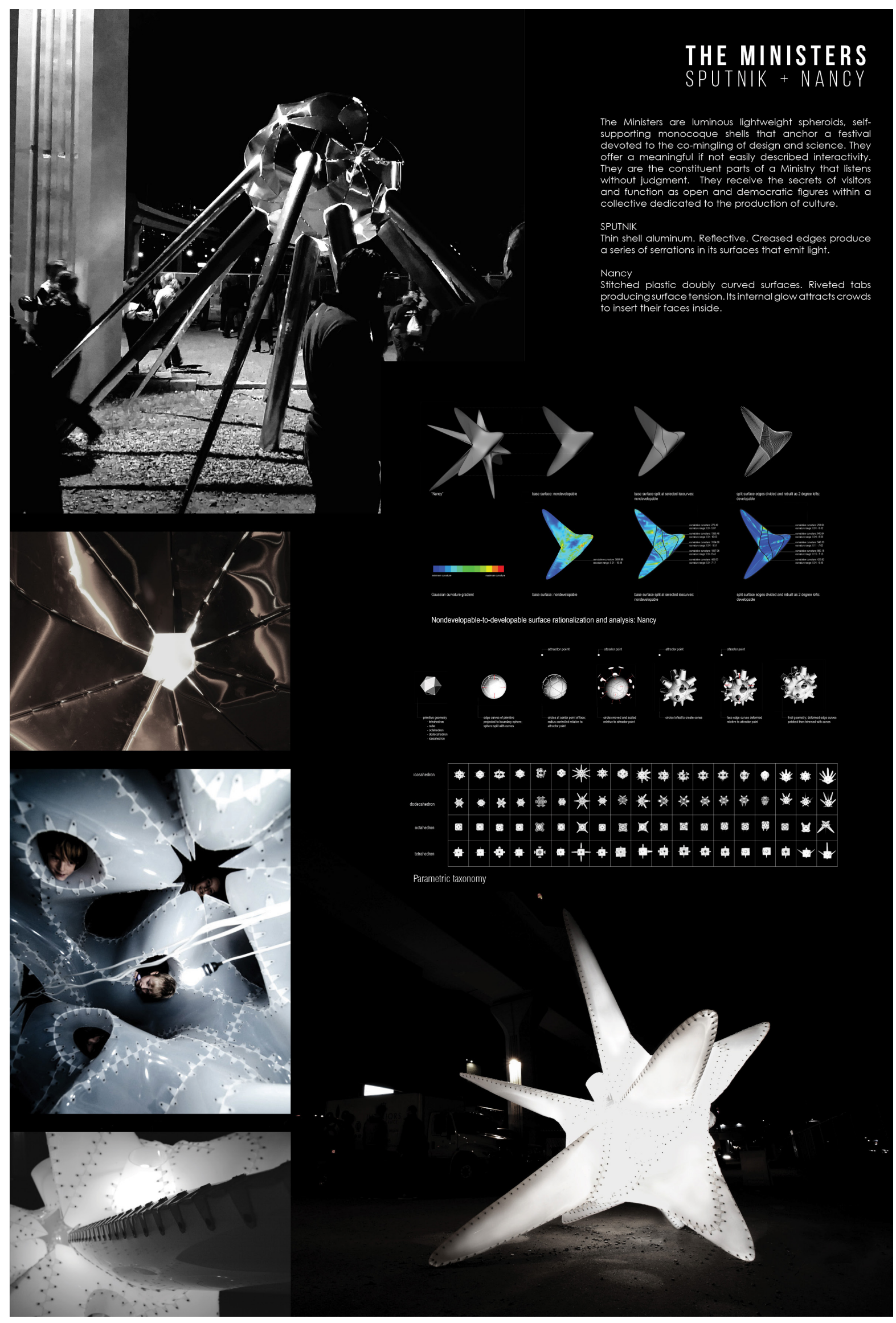

